EPJ Web of Conferences 45, 01120 (2013)

DOI: $10.1051 /$ epjconf/20134501120

(C) Owned by the authors, published by EDP Sciences, 2013

\title{
Identification of collapse patterns of cavitation bubbles close to a solid wall
}

\author{
M. Müller ${ }^{1}$, J. Hujer $^{1}$, M. Kotek ${ }^{2}$ and P. Zima ${ }^{3}$ \\ ${ }^{1}$ Technical University of Liberec, Faculty of Mechanical Engineering, 46117 Liberec 1, Czech Republic \\ ${ }^{2}$ Technical University of Liberec, Faculty of Mechatronics, Informatics and Interdisciplinary Studies, 46117 Liberec 1, \\ Czech Republic \\ ${ }^{3}$ Institute of Thermomechanics ASCR, v. v. i., Department of Thermodynamics, 18200 Prague 8, Czech Republic
}

\begin{abstract}
The article describes different patterns of bubble deformation during the cavitation bubble collapse close to a solid wall for different bubble-wall distances. The bubble is generated by energy discharge in water. The collapse patterns are investigated using high-speed photography. The magnitude of the bubble-wall interaction is measured using the PVDF film. The shock wave pressure in the far field is measured using the PVDF hydrophone.
\end{abstract}

\section{Introduction}

The careful determination of the type of interaction between the cavitation bubble and the solid wall during the bubble collapse is important in order to estimate the damage potential of the collapsing bubble. It is generally accepted (for example [1]) that the cavitation damage is caused by three main actions: i) the fast liquid microjet formed immediately after the collapse and directed towards the solid wall; this effect is significant for bubbles at medium distance from the wall, ii) the splashing effect, which is significant for bubbles in direct contact with the wall, or iii) the shock wave [1], [2], which is the main form of interaction for bubbles collapsing far from the wall.

The aim of this study is to demonstrate the different collapse patterns and offer methodology to identify them. Each collapse pattern is observed by the high-speed camera and related to the pressure signal measured by the needle hydrophone located in far field and the impact force measured by the PVDF film attached to the wall at the position of the collapse-induced impact. The two measurements enable to identify the bubble collapse patterns without optical observation.

The typical voltage signal from the hydrophone is shown in figure 1 . The first half of the signal corresponds to the bubble growth to the first maximum radius and the first collapse. The oscillatory second half corresponds to the bubble growth to the second maximum radius and the second collapse. Previous works [3], [4] have shown that the ratio between the magnitude of the first and second collapses depends on the type of collapse pattern (which will be discussed later). The peaks in the signal reveal the violent character of the collapses. Therefore, to achieve satisfactory temporal resolution of the collapse phenomena the resolution in the nanosecond range is required.

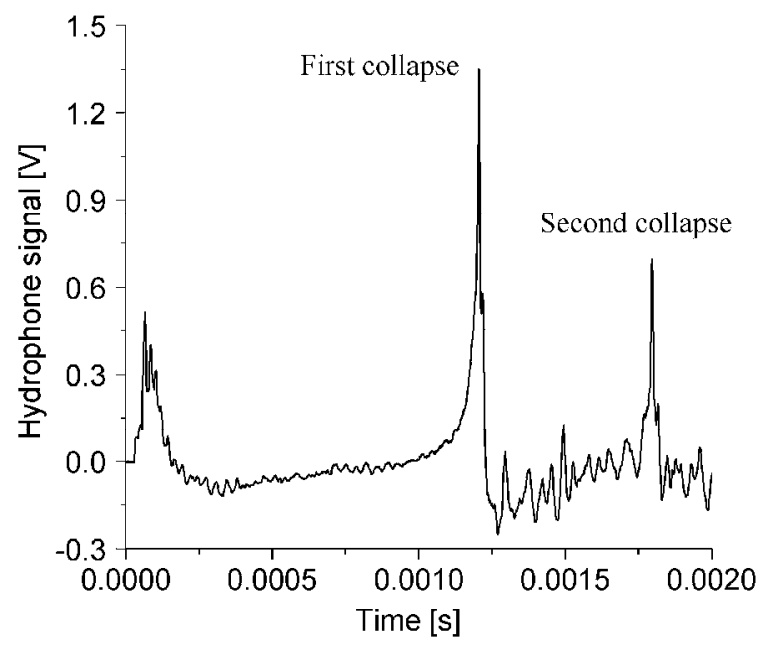

Fig. 1. Typical voltage signal of cavitation bubble collapsing in the liquid measured by PVDF hydrophone.

In this study the bubble is generated by a low voltage discharge into crossing wires [3], [5]. This technique provides a simple way of producing spherical bubbles of different diameters. The disadvantage of the 
method is that the minimum bubble size (i.e. the maximum bubble diameter after the first expansion) is determined by the diameter of the generating wires. The wires are initially in direct contact with each other so that no plasma is generated during the bubble initiation.

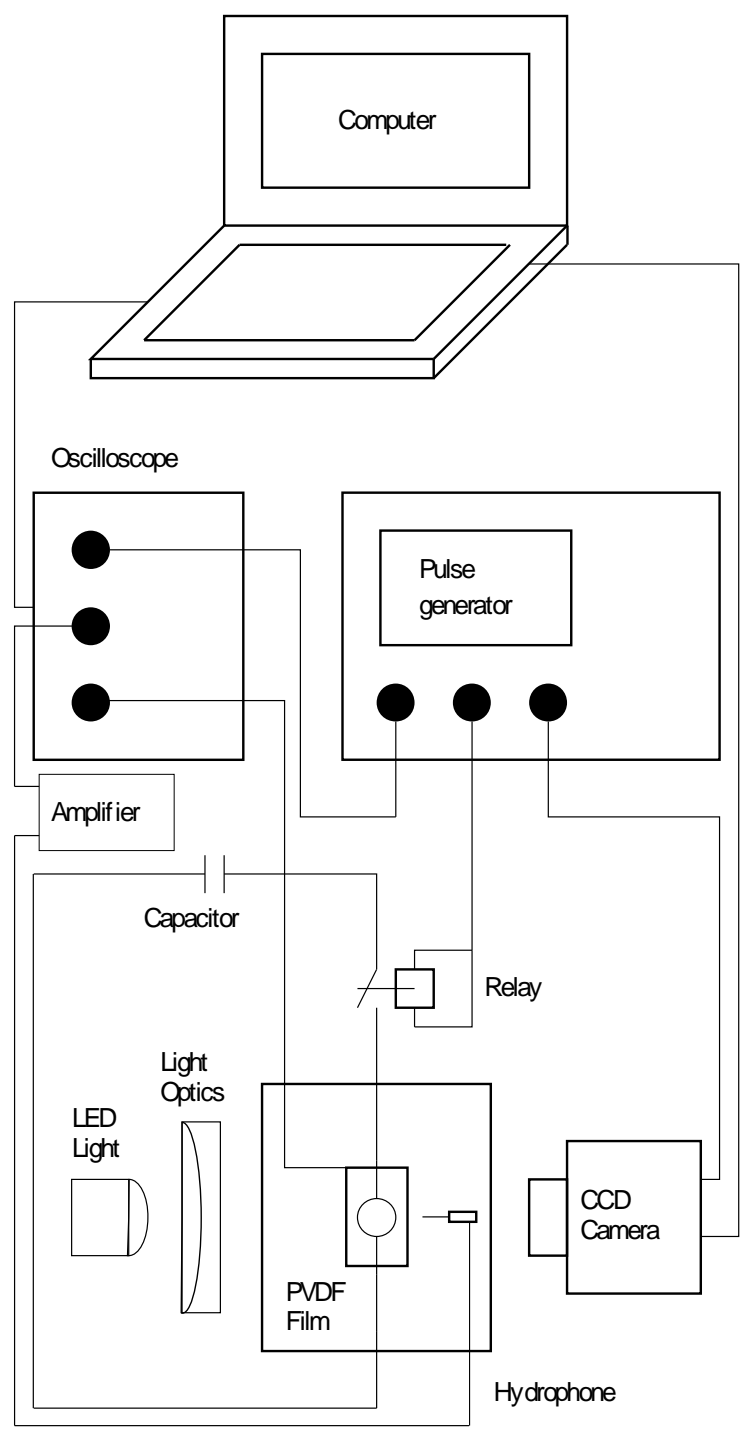

Fig. 2. Experimental setup used in the investigation of the cavitation bubble collapse.

\section{Experimental setup}

The experimental setup for the investigation of a single spark-generated cavitation bubble is shown in Figure 2. The bubble is created between two copper electrodes (thin wires of diameter $0.19 \mathrm{~mm}$ ) placed in a glass bath filled with distilled water. The electric conductivity of the water is monitored and maintained in the range $3-11 \mu \mathrm{S} / \mathrm{m}$ during the experiment. The electrodes are in direct contact to enable the use of low voltage. The spark is produced by two parallel capacitors $(4700 \mu \mathrm{F})$ discharged through a relay. The temporal evolution of the bubble radius was recorded using the high-speed Phantom CCD camera with 64,000 fps. The illumination is provided by the power LED diode (Rapp OptoElectronic). PVDF film sensors DT1-028K W/TH (Measurement Specialties) are used to record the impact force generated by the bubble collapse. The pressure response in the far field is measured using the PVDF needle hydrophone (RP-Acoustic). The hydrophone is mounted in the same movable frame as the electrodes so that the distance between the bubble origin and the hydrophone is kept constant $(1.5 \mathrm{~cm})$. The tip of the PVDF hydrophone is visible in the upper part of the photographic images (for example, in Figure 3) whereas the PVDF film can be seen at the bottom of the images. The signals are digitalized using the oscilloscopic card with the sampling frequency $60 \mathrm{MHz}$. The PVDF film is calibrated using the drop ball test described in [3], and the measured calibration constant is $7.48 \mathrm{mV} / \mathrm{N}$.

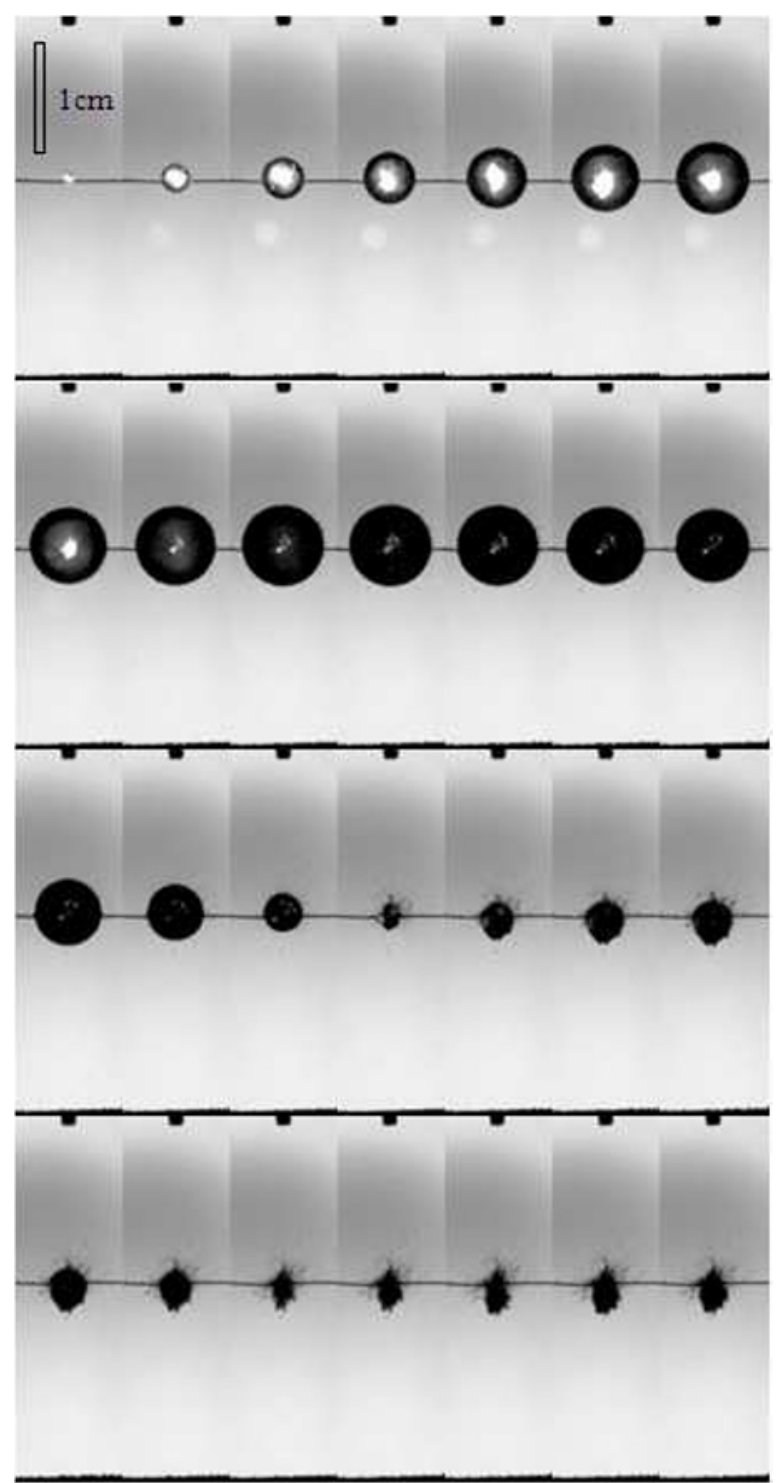

Fig. 3. Collapse pattern 1 - high-speed camera record. Time distance between the frames is $62.5 \mu \mathrm{s}$. 


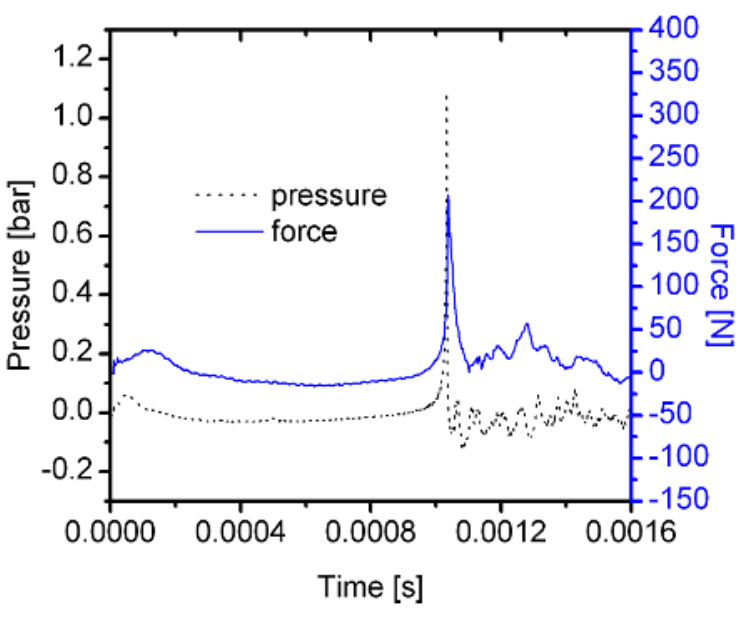

Fig. 4. Collapse pattern 1 - hydrophone and PVDF film signals.

\section{Results}

Figures 3 and 4 show the bubble collapse pattern typical for bubbles located far from the wall. In this case, the bubble dynamics is not influenced by the wall presence and bubble growth and collapse are almost spherical. The force generated by the first collapse and measured by the PVDF film is much higher than the force generated by the second collapse. The liquid microjet is not formed. This collapse pattern is characterized by the strong first collapse. The interaction between the bubble and the wall is realized by the shock wave propagated from the bubble origin.

Figures 5 and 6 show the bubble collapse pattern typical for bubbles at medium distance from the wall, which do not touch the wall during their first expansion. In this case, the bubble shape and dynamic behaviour is influenced by the presence of the wall. The bubble is attracted to the wall due to accelerated flow between the bubble and the wall. The magnitudes of the first and second collapses (i.e. the impact forces and pressures seen as peaks in figure 6) are comparable. Liquid microjet directed to the wall is generated immediately after the first collapse (see figure 5). As the bubble moves to the wall the second collapse occurs at a closer distance than the first collapse. As a result, the magnitude of the second collapse is comparable to the first collapse. The subsequent collapses occur directly at the wall, however, their magnitude is small as most of the bubble energy is dissipated by the first two collapses.

Figures 7 and 8 show the third type of cavitation bubble collapse pattern. The bubble is in direct contact with the wall during the first expansion. This is apparent from the PVDF film signal in Figure 8 as significant amount of force is exerted on the PVDF film during the growth phase. The first collapse occurs very at the wall and the bubble is sucked to the wall. The bubble breaks into a complex non-spherical structure after the first collapse. During the second collapse the structure is already firmly attached to the wall and the collapse occurs at the wall. Due to the sucking effect the magnitude of the pressure peak from the second collapse is higher than the magnitude of pressure peak from the first collapse. The impact force signal from the PVDF film is more complex as the splashing effect generates a significant amount of force acting on the wall between the collapses. This collapse pattern is characterized by the higher magnitude of the second collapse than the first collapse and a significant amount of force on the wall during the bubble expansion (first as well as second).

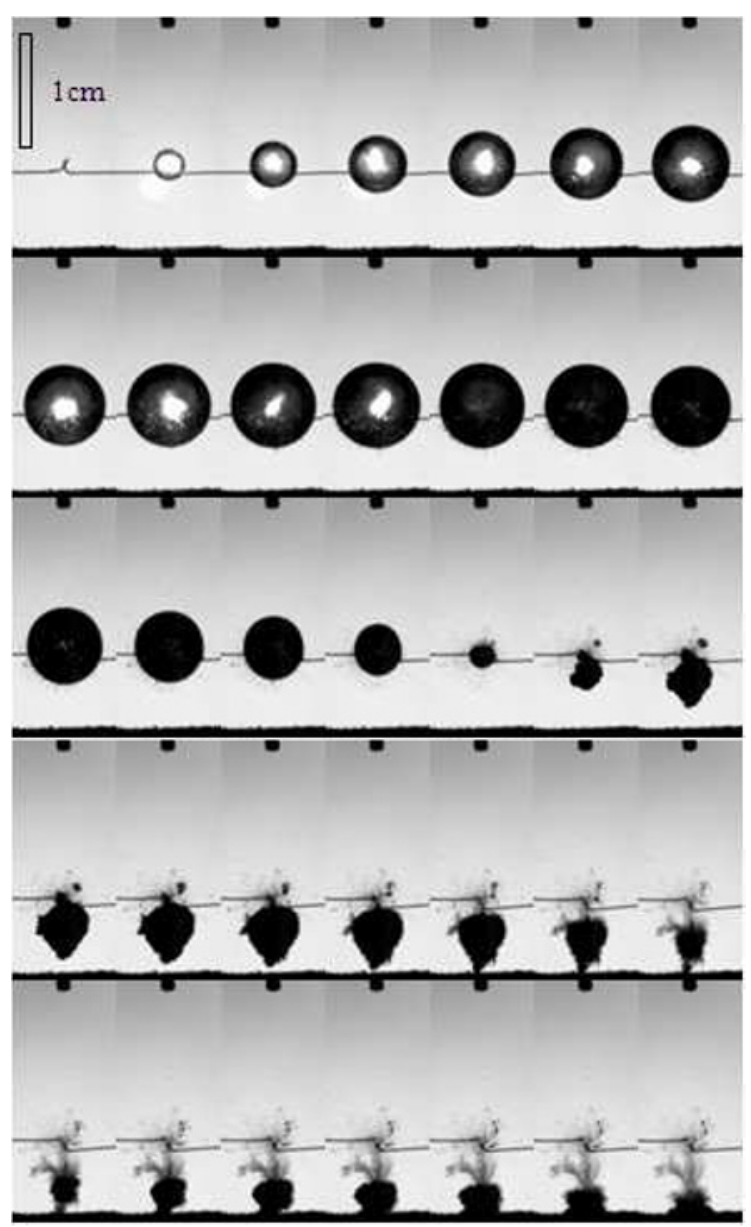

Fig. 5. Collapse pattern 2 - high-speed camera record. Time distance between the frames is $62.5 \mu \mathrm{s}$.

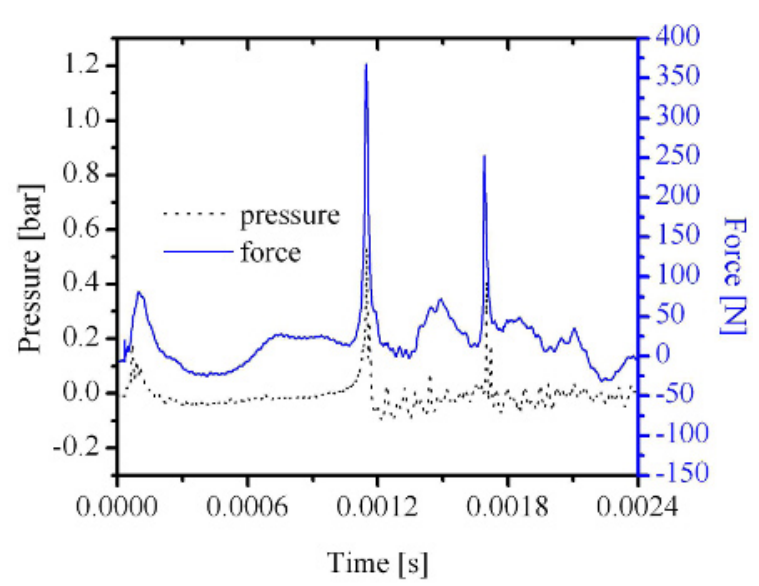

Fig. 6. Collapse pattern 2 - hydrophone and PVDF film signals. 
It should be noted that for all the above measurements the PVDF hydrophone only detects acoustic signals emitted by the bubble and therefore it is mainly a measure of the strength of the shock wave emitted during the bubble collapse rather than the mechanical action on the wall. The combined action of the acoustic pressure and the liquid flows acting on the wall is represented by the PVDF film signal.

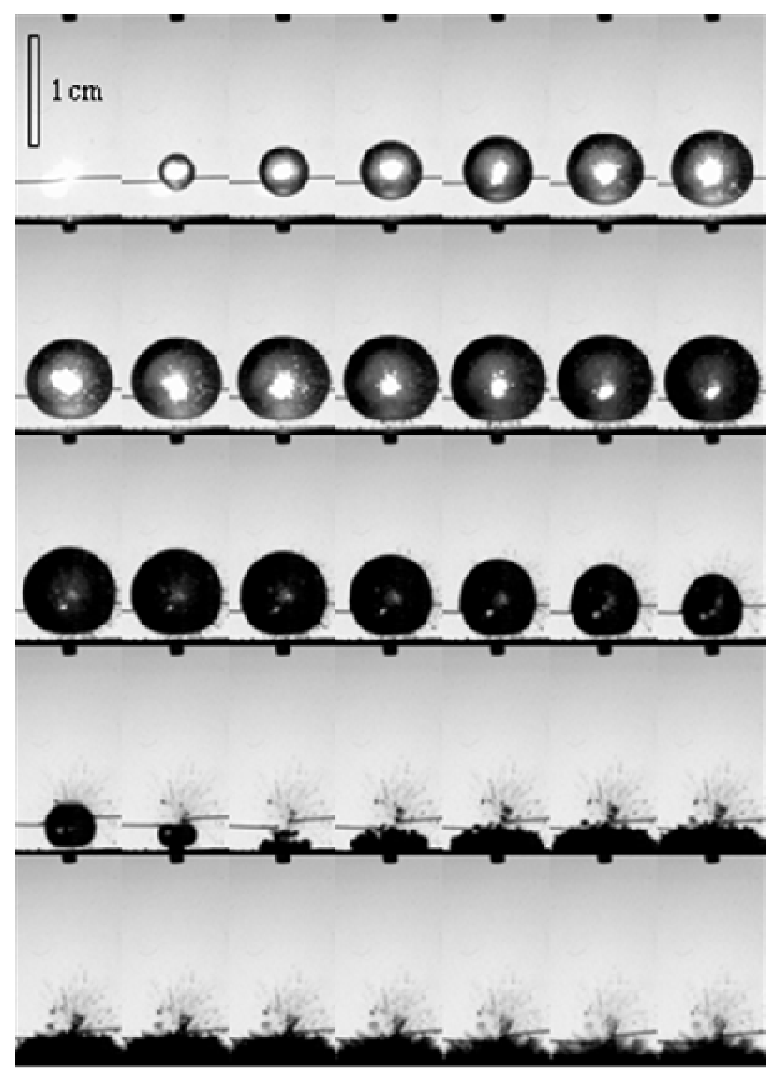

Fig. 7. Collapse pattern 3 - high-speed camera record. Time distance between the frames is $62.5 \mu \mathrm{s}$.

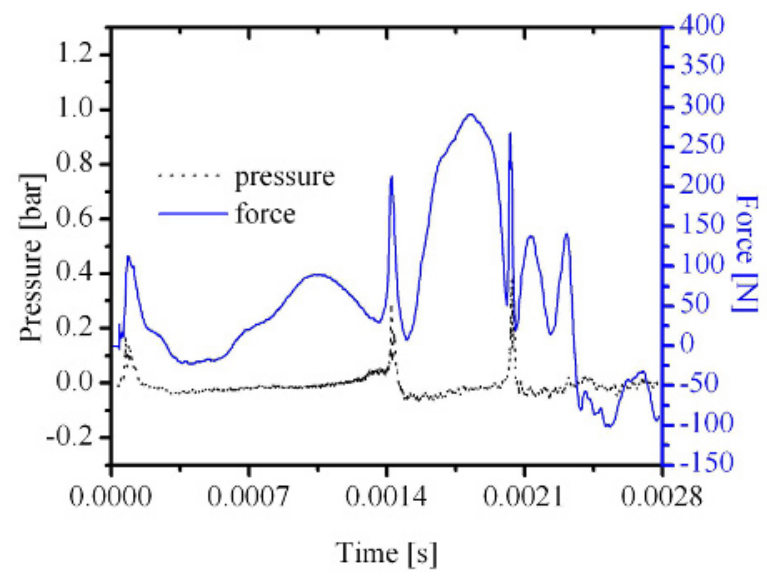

Fig. 8. Collapse pattern 3 - hydrophone and PVDF film signals.

\section{Conclusion}

The cavitation bubble collapse patterns for bubbles collapsing close to a solid wall were investigated by acoustical and optical methods. Three different collapse patterns were observed from the measurement. The first collapse pattern occurs for bubbles collapsing far from the wall. The magnitude of the first collapse is significantly higher than the magnitude of the second collapse. The second collapse pattern is characterized by the similar magnitude of the first and the second collapse. This pattern occurs when the bubble collapses close to the wall. The third collapse pattern occurs when the bubble is collapsing directly at the wall. The magnitude of the second collapse is usually higher than the magnitude of the first collapse.

The method of identification of the three collapse patterns was presented. It is based on careful examination of the measurement signals from the PVDF film attached to the wall and the PVDF hydrophone in the far field. It allows us to determine the type of collapse pattern without optical observation of the bubble collapse.

The further objective of the authors is to quantify the limits between the three different collapse patterns using the maximum bubble radius and the difference of the bubble origin from the wall.

\section{Acknowledgements}

This work was made possible by grant no. 101/10/1428 of the Czech Science Foundation, partly by the project "OP VaVpI Centre for Nanomaterials, Advanced Technologies and Innovation“ (CZ.1.05 /2.1.00/01.0005), and by the project "Development of Research Teams of R\&D Projects at the Technical University of Liberec" (CZ.1.07/2.3.00/30.0024).

\section{References}

1. C. E. Brennen, Cavitation and Bubble Dynamics, Oxford University Press, Oxford (1995)

2. J. P. Franc, J. M. Michel, Fundamentals of cavitation, Kluwer Academic Publisher, New-York (2004)

3. M. Müller, P. Zima, J. Unger, Energy dissipated during the cavitation bubble collapse close to a solid wall, Proceeding of CAV2009, 7th International Symposium on Cavitation, Paper No. 259, Singapore (2012)

4. Y. C. Wang, Y. W. Chen, Application of piezoelectric PVDF film to the measurement of impulsive forces generated by cavitation bubble collapse near a solid boundary, Exp. Thermal Fluid Sci. 32, 403-412 (2007)

5. L. W. Chew, E. Klaseboer, S. W. Ohl, B. C. Khoo, Interaction of two differently sized oscillating bubbles in a free field, Physical Review E. 84, 066307 (2011) 